\title{
MPCSL - A Modular Pipeline for Causal Structure Learning
}

\author{
Johannes Huegle* \\ johannes.huegle@hpi.de \\ Hasso Plattner Institute, University of Potsdam \\ Potsdam, Germany \\ Michael Perscheid \\ michael.perscheid@hpi.de \\ Hasso Plattner Institute, University of Potsdam \\ Potsdam, Germany
}

\author{
Christopher Hagedorn* \\ christopher.hagedorn@hpi.de \\ Hasso Plattner Institute, University of Potsdam \\ Potsdam, Germany \\ Hasso Plattner \\ hasso.plattner@hpi.de \\ Hasso Plattner Institute, University of Potsdam \\ Potsdam, Germany
}

\begin{abstract}
The examination of causal structures is crucial for data scientists in a variety of machine learning application scenarios. In recent years, the corresponding interest in methods of causal structure learning has led to a wide spectrum of independent implementations, each having specific accuracy characteristics and introducing implementation-specific overhead in the runtime. Hence, considering a selection of algorithms or different implementations in different programming languages utilizing different hardware setups becomes a tedious manual task with high setup costs. Consequently, a tool that enables to plug in existing methods from different libraries into a single system to compare and evaluate the results is substantial support for data scientists in their research efforts.

In this work, we propose an architectural blueprint of a pipeline for causal structure learning and outline our reference implementation MPCSL that addresses the requirements towards platform independence and modularity while ensuring the comparability and reproducibility of experiments. Moreover, we demonstrate the capabilities of MPCSL within a case study, where we evaluate existing implementations of the well-known PC-Algorithm concerning their runtime performance characteristics.
\end{abstract}

\section{CCS CONCEPTS}

- Mathematics of computing $\rightarrow$ Causal networks; $•$ Computing methodologies $\rightarrow$ Causal reasoning and diagnostics; • General and reference $\rightarrow$ Evaluation; Experimentation.

\section{KEYWORDS}

Causal Structure Learning, Modular Pipeline, Evaluation Framework, Benchmarking

\section{ACM Reference Format:}

Johannes Huegle, Christopher Hagedorn, Michael Perscheid, and Hasso Plattner. 2021. MPCSL - A Modular Pipeline for Causal Structure Learning. In Proceedings of the 27th ACM SIGKDD Conference on Knowledge Discovery

${ }^{*}$ Both authors contributed equally to this research.

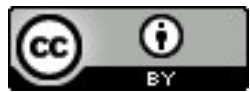

This work is licensed under a Creative Commons Attribution International 4.0 License. KDD '21, August 14-18, 2021, Virtual Event, Singapore.

(C) 2021 Copyright held by the owner/author(s).

ACM ISBN 978-1-4503-8332-5/21/08

https://doi.org/10.1145/3447548.3467082 and Data Mining (KDD '21), August 14-18, 2021, Virtual Event, Singapore. ACM, New York, NY, USA, 9 pages. https://doi.org/10.1145/3447548.3467082

\section{INTRODUCTION}

The knowledge about the causal structures between the variables of a system is of high interest for data scientists and researchers in a variety of domains [31]. Examples are drug design, where Causal Graphical Models (CGMs) derived from gene expression data depict genetic regulatory relationships [34] or manufacturing, where the knowledge about causal structures supports the root cause analysis of failures within complex production processes, e.g., see [18].

In this context, the growing interest in methods of Causal Structure Learning (CSL) has lead to a wide spectrum of scientific publications, each tackling different domain-specific constraints, e.g., statistical considerations to improve the accuracy in non-linear settings [45] or the examination of parallel computing strategies [24, $37,39]$ or adoption of accelerator cards $[38,48]$ to speed-up the learning process. This lead to the development of a number of libraries in different programming languages [19, 22, 24, 36, 39, 46] or standalone implementations [37, 38, 48] each introducing implementation-specific overhead to both the accuracy of the results and the runtime of CSL. Therefore, data scientists of different domains are confronted with the omnipresent challenge of selecting their most appropriate algorithm for CSL given the unique characteristics of their domain. This may be accompanied by specific hardand software constraints or unique data characteristics. In these scenarios, determining both the correct algorithmic approach with the corresponding choice of the algorithm's parameters and the most suitable implementation for a given observational dataset becomes cumbersome. Even though studies comparing algorithms for CSL exist and provide an indication for the best choice [41], they are limited to the algorithms considered and available at the time of writing, as well as the data characteristics utilized in the experimental evaluation. Hence, the evaluation of experiments given an observational dataset, considering a selection of state-of-the-art algorithms or different implementations in different programming languages utilizing different hardware setups becomes a tedious manual task with high setup costs. In particular, in real-world scenarios, both missing ground truth and the computational complexity further impede the evaluation of the algorithm's reliability, e.g., see [11,21]. For example in genetics, large-scale gene expression data introduces domain-specific constraints with regard to statistical assumptions on the complex mostly unknown underlying biological mechanisms 


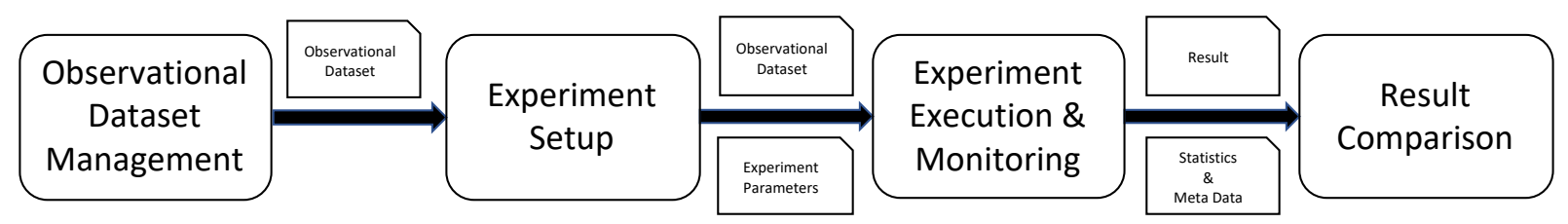

Figure 1: The essential components of the modular pipeline for CSL required for a comprehensive benchmarking of different CSL algorithm implementations given observational datasets.

on one side and algorithms' performance required for CSL in this high-dimensional setting on the other side $[1,10]$. Therefore, for biologists aiming to derive gene regulatory networks, the question of practical relevance is the appropriate choice of an existing method for CSL for their genetic observational data set specific to their biological research question. As the vastly expanding field of research in methods of CSL results in a "sheerly unlimited number of methods" neither is a complete evaluation feasible [10] nor is the computational complexity manageable without an interdisciplinary team that incorporates computer scientists, too [1].

Moreover, data scientists working on advancements of existing algorithms for CSL are confronted with the task of considering both naive baseline methods and novel competitive methods in their evaluation. For example in the research field of hardware acceleration in CSL, a comprehensive evaluation becomes a tedious manual task with high setup costs as it requires the orchestration of a setup that incorporates not only different programming languages such as $\mathrm{R}, \mathrm{C}++$, or Python but also experiments in a heterogeneous hardware setup with multi-core CPUs and GPUs [38].

Consequently, the access to a tool that enables to plug in existing methods from different libraries into a single system to compare and evaluate the results, also under the prism of improving existing algorithms, is of great importance. In the machine learning domain, such tools follow a methodology referred to as the Common Task Framework (CTF) [8], which has the following three components: publicly available training datasets, a set of competitors, and a scoring referee to compare the competitors. For the challenge of incorporating and evaluating existing methods and implementations for CSL, we introduce a pipeline structure as depicted in Figure 1. Starting with an Observational Dataset Management and given an Experimental Setup, which enables the selection of both appropriate algorithms, and execution environments, the pipeline needs to orchestrate the experiments in a step for Experiment Execution $\&$ Monitoring. To evaluate the results of CSL with regard to both the runtime performance, and the accuracy of the results a Result Comparison component completes the pipeline.

The variety of algorithms, different hardware requirements, and constraints in a scientific research setting imply the following requirements towards a platform-independent, modular pipeline that ensures the comparability and reproducibility of experiments.

Modularity: The pipeline should be implemented based on the principle of modularity [30] to enable easy adaptation for extensions of the components' feature set, e.g., the addition of new metrics for comparing CSL algorithms in different dimensions to support a wide range of evaluation objectives.
Platform Independence: Applying concepts of virtualization and containerization, the pipeline should be designed in a platformindependent manner to support various implementations, independent from the programming language or the hardware requirements, in a single pipeline.

Comparability and Reproducibility: In order to address the high scientific demand of the research community, all metadata, which contains information regarding the experimental setup, e.g., dataset, algorithms' parameters, or information regarding the underlying hardware setup, should be persisted.

Following the previously introduced requirements for a modular CSL pipeline, our contributions are: First, the derivation of a blueprint for a modular CSL pipeline to support data scientists in their research on methods for CSL. Second, the provision of a reference implementation, called MPCSL, accessible to the research community. Third, a case study to demonstrate the applicability of MPCSL determining suitable CSL implementations regarding runtime for a range of observational datasets.

The remainder of the paper is organized as follows. After providing an overview of the theoretical background on CSL in Section 2, we discuss existing approaches to benchmark and compare causal structure learning algorithms in Section 3. In Section 4, we present a blueprint of a pipeline that meets the previously introduced requirements and provide detail on our reference implementation. The capabilities of our presented pipeline are demonstrated in Section 5, in which we evaluate existing implementations of the well-known PC-Algorithm with regard to their runtime in a case study. We summarize our work in Section 6.

\section{CAUSAL STRUCTURE LEARNING}

In this section, we sketch the theoretical background as well as the main approaches of CSL. For more information and a more detailed introduction we refer to the comprehensive work of Pearl [31], Spirtes et al. [44], or Peters et al. [32].

\subsection{CGM and Causal Inference}

Causal Graphical Models (CGMs) are a recognized fundamental concept of the formalization of causality [15, 31, 44]. In this context, a CGM $\mathcal{G}=\{\mathbf{V}, \mathbf{E}\}$ encodes the causal relationships over a set of random variables $\mathrm{V}$, in the sense that any edge $X \rightarrow Y$ in $\mathbf{E}$ between the variables in $\mathrm{V}$ represents a direct causal effect. The directed edges in $\mathcal{G}$ entail the causal interpretation that variable $Y$ is a function of its direct cause $X$ and some noise term $\epsilon$, i.e., $Y=f(X, \epsilon)$, where $\epsilon$ is independent of $X$. In other words, it says that for an edge $X \rightarrow Y$ in the CGM $G$ an intervention on $X$ will directly change the distribution or value of $Y$. For example 
let $X$ and $Y$ be directly causally influenced through a common confounder $Z$, i.e., $X \leftarrow Z \rightarrow Y$. Observing associations in changes on $X$ and $Y$ induced through the common confounder may let you falsely assume that a causal relationship exists. While changes through interventions on $Z$ will directly change both $X$ and $Y$ neither interventions in $Y$ nor in $X$ will affect $Z$ or $Y$, respectively.

Following this idea, Judea Pearl introduced the $d o(\cdot)$-operator into the framework of CGMs as a notion to distinguish the observational conditional probability $P(Y \mid X=x)$, describing the probability distribution of $Y$ if one observed $X=x$, from the conditional interventional probability $P(Y \mid d o(X=x))$ that depicts the probability distribution of $Y$ when $X$ has been set to $x$ [31].

Traditionally, a causal relationship has been discovered through the execution of randomized experiments or interventions into the system itself, which may be too expensive, time-consuming, or even impossible [11]. Hence, the framework of CGMs in cooperation with the $d o(\cdot)$-operator enables a comprehensive examination of causal structures on the basis of observational data [32].

\subsection{Assumptions and Approaches of CSL}

Causal structure learning can be characterized as the task of deriving as much of the underlying causal relationships in the CGM $\mathcal{G}$ from observational data as possible. Therefore, the CGM needs to be connected to the joint probability distribution of its variables which comes with constraints on the structures or the corresponding functional system. Naturally, the CGM is assumed to be acyclic, i.e., there is no feedback loop in $\mathcal{G}$, and causal sufficiency, i.e., there is no latent confounding variable affecting the variables in $\mathcal{G}$. Moreover, the principles of causal Markov assumption, causal faithfulness and causal sufficiency enable to connect the CGM $\mathcal{G}$ with probabilistic characteristics of the joint probability distribution of the corresponding variables [31]. The high-level idea is that these assumptions imply a corresponding conditional independence structure in the joint probability distribution and the causal structures within the CGM $\mathcal{G}$ via the so-called $d$-separation criterion.

Algorithms for learning the causal structures build upon these assumptions to leverage the probabilistic characteristics of the observational dataset and can be categorized into score-based, constraintbased, or hybrid approaches. Constraint-based CSL derives the information about probabilistic characteristics within the observational dataset through the repeated application of conditional independence (CI) tests in a first step. Given the dataset's probability distribution, different $\mathrm{CI}$ tests can be plugged-in within this iterative procedure that estimates the undirected skeleton of $\mathcal{G}$. In a second step, deterministic rules are applied to orient the undirected edges. One of the most popular constraint-based algorithms is the PCAlgorithm by Sprites et al. [44] together with its order-independent version PC-Stable [5]. Given reliable CI tests, constraint-based algorithms are flexible and able to handle various data distributions which make them widely applicable [11]. Yet, the long execution time poses a challenge for its application in practice, in particular for high-dimensional data [23]. Algorithms following the scorebased approach evaluate a score on all possible Directed Acyclic Graphs (DAGs) in relation to the data, typically using a penalized likelihood score, and search for the DAG that yields to the optimal score [3]. As the search space of possible DAGs is too large, greedy approaches are used but still raise an NP-hard problem [4]. Greedy Equivalence Search is a well-known greedy two-phase procedure for score-based CSL [3]. Hybrid algorithms, such as the Max-Min Hill Climbing algorithm [47], are a combination of both previously introduced approaches. In the first step, CI tests are used to limit the search space for the subsequent application of a score-based approach to finding the DAG with a maximal score.

Note that for all approaches exist a variety of modifications and extensions that can handle a weakened set of assumptions to enable a wider range of applications. For example in the presence of latent confounders, i.e., a violation of causal sufficiency, methods such as the constraint-based FCI algorithm [44] or the hybrid GFCI algorithm [29] provide asymptotic correct results.

\section{RELATED WORK}

The challenge of comparing and evaluating a selection of algorithms is omnipresent in the research community, and requires an objective evaluation of competitors, as portrayed in the Common Task Framework (CTF) [8]. In the area of causality in general, Dorie et al. [9] implemented the idea of a common task in the "2016 Atlantic Causal Inference Competition”, the first large-scale data analysis competition for causal inference from observational data. Though the results explore the relative strengths and weaknesses of methods for causal effect estimation, a sound causal inference requires knowledge about the causal structures of the problem [17]. Hence, causal competitions should evaluate the entire causal inference pipeline incorporating both the examination of methods for causal structure learning and for effect estimation [20].

There exist several attempts to provide tools for the empirical evaluation of methods for causal effect estimation to support an objective comparison between competitors. For example, Lin et al. [25] propose an API for evaluating causal inference models inspired by the idea of CTF aiming to provide a centralized competition platform for and extended by researchers in causal inference. The researcher can submit new models, datasets, metrics, and parameterizations, which become available to the community and allow for more comparable benchmarks. Shimony et al. [43] present a causal inference benchmarking framework distributed on github.com to support a better comparison between methods that estimate causal effects.

In contrast to the task of evaluating methods for causal effect estimation, to the best of our knowledge, there is no common evaluation framework for causal structure learning algorithms across multiple programming languages. Within the R programming language [33] the package CompareCausalNetworks [16] provides an interface to compare supported causal structure learning algorithms from different classes. In particular, the authors of the package evaluate, PC [44], FCI [44], GES [3], GIES [14], MMHC [47], LINGAM [42] and backshift [35] on simulated graphical networks and use the relations isAncenstor and isParent to compare the learned causal structures across the algorithms. The study provides an overview of the applicability of the methods for researchers, yet it is limited to the considered data characteristics, i.e., not covering any datasets above 100 variables. Furthermore, the package only supports algorithms written in the R-Framework, not supporting implementations written in other programming languages. 
A range of different libraries written in $\mathrm{R}$ try to provide a toolbox for causal structure learning, e.g., pcalg [19], or bnlearn [39]. Yet, neither do they support the data scientist with regard to a comparison of runtime performance and accuracy of the results, nor do they cover all existing algorithms. For example, smaller packages in various programming languages implement research in the context of improvements within different statistical settings, e.g., kpc [45] for non-linear causal relationships, or in the context of algorithmic advances for improved runtime performance, e.g., parallelPC [24] or Lock-Free-PC [37] addressing parallel execution strategies, or cupc [48] for execution on accelerator cards. Each library covers a mix of the available approaches and implements particular algorithms in their respective programming language. Hence, a comparison to other implementations for CSL becomes a tedious manual task with high setup costs. While there are software tools, whose construction follows the pipeline structure of Figure 2 to support a comparison of results, e.g., BayesiaLab [6] or tetrad [36], they are limited to the algorithms implemented within the respective software tools.

With our pipeline, we provide a framework to incorporate existing libraries and thereby compare implementations across multiple libraries and various programming languages, without the need to manually change the experimental setup for each implementation.

\section{A MODULAR PIPELINE FOR CSL}

In this section, we present a modular pipeline for Causal Structure Learning (CSL) that addresses the requirements on platform independence and modularity while ensuring the comparability and reproducibility of experiments. We propose an architectural blueprint and outline our reference implementation ${ }^{1}$.

\subsection{An Architectural Blueprint}

In the following, we introduce an architectural blueprint, depicted in Figure 2, for a modular pipeline that covers the necessary steps for benchmarking algorithms of CSL. It is designed to fulfill the prior mentioned requirements and consists of four main elements. A Web Application provides visual support to a data scientist, and communicates with the core of the pipeline, the Backend Service. A separate service, the Experiment Execution Service, is responsible for the creation, scheduling, and monitoring of dedicated Execution Containers. These Execution Containers run specific causal structure learning algorithms and derive the CGMs based on the observational data, which are stored within the Backend Service.

Web Application: The Web Application provides an interactive front-end that supports the data scientist in the task of creating the experimental setup following the pipeline paradigm depicted in Figure 1. The Web Application communicates with the Backend Service to create the user-defined experimental setup which includes the source of the dataset, the method of CSL with the corresponding library, and implementation-specific parameters of the algorithm. For an experiment, multiple experiment runs can be executed, and thereupon monitored in the Web Application to ensure flawless execution. Once one execution is finished, the Web Application provides mechanisms for the data scientist to explore the determined causal relationships, e.g., visualizing the CGM, examining

\footnotetext{
${ }^{1}$ https://github.com/hpi-epic/mpcsl
}

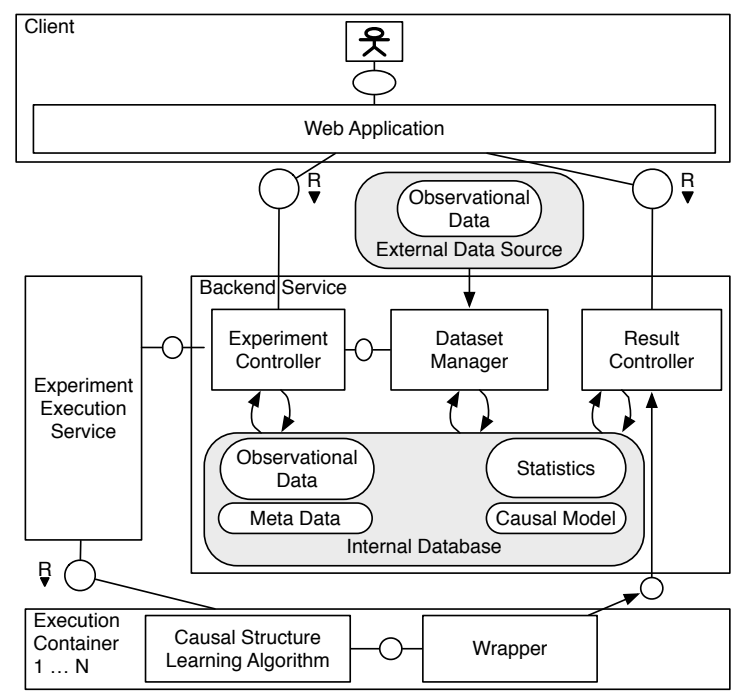

Figure 2: Architectural blueprint of a modular pipeline for causal structure learning.

data distributions, causal dependencies, or causal effect estimation using the do-operator [31]. Further, the Web Application visualizes the collected execution statistics, e.g., runtimes of the selected CSL implementation, and enables to examine predefined accuracy metrics of the learned CGM, e.g., the false positive rate of edges with respect to the expected ground truth graphical model.

Experiment Execution Service: The Experiment Execution Service is responsible for the execution of experiment runs. Upon request by the Backend Service, it creates Execution Containers according to the requirements defined for the experiment, e.g., fitting to the CSL implementation, and on the requested available hardware. Using independent Execution Containers, which are scheduled on the appropriate hardware, ensures platform independence. The experiment runs are scheduled and according to the setup, the Experiment Execution Service ensures sequential execution to avoid the influence between different experiments. Further, the experiment runs are monitored and the information is provided to the Backend Service.

Backend Service: The Backend Service handles the requests posted by the Web Application. Therefore, it communicates with the Experiment Execution Service, stores metadata for experiments, e.g., parameter settings and information on datasets to ensure reproducibility and comparability of experiment runs. The Backend Service is composed of a Dataset Manager, an Experiment Controller, an Internal Database and a Result Controller.

The Dataset Manager handles all internal and external requests related to the datasets, i.e., creation, deletion, or access. During the creation of a dataset, its metadata, i.e., an identifier, a name and a description of the dataset, the data source, an access method, and a validity flag, and a list of its variables are stored within the Internal Database of the Backend Service. Moreover, a dataset is invalidated in case of critical changes revoking any new experiment to ensure comparability and reproducibility. The data source points to the 
location the observational data is stored, i.e., the Internal Database or an External Data Source, including access information such as an SQL select statement to retrieve the data.

The Internal Database stores the above-described metadata on all observational datasets, regardless of their source. Further, it stores the available implementations for CSL within the pipeline, the parameter setup of all created experiments together with the learned CGM and corresponding statistics collected during execution. In combination with the information on the utilized hardware, stored for each experiment run, comparability and reproducibility of results are ensured.

The Experiment Controller handles requests from the Web Application concerning experiments and their setup, i.e., registration of observational datasets, creation of experiments, or triggering experiment runs. Registration of observational datasets and obtaining observational datasets for experiment execution are forwarded to the Dataset Manager. The corresponding metadata on the experiments is queried and send, together with samples from the observational dataset to the Experiment Execution Service in case an experiment run is triggered. After execution, the Result Controller receives the learned CGM and collected statistics from experiment runs through the Wrapper available within the Execution Containers. Further, the Execution Container enables an evaluation of the experiment results through the calculation of accuracy metrics with respect to the learned CGM in comparison to the ground-truth stored in the Internal Database. Note, the modular extensibility ensures the incorporation of more advanced accuracy metrics beyond false-positive rates, graph edit distances, or the Structural Hamming Distance (SHD) [47] that may be required within the respective experimental evaluation.

Execution Containers: The Execution Containers are orchestrated by the Backend Service and provide a virtualized environment for the execution of algorithms for CSL. Thus, appropriate execution environments according to the requirements of the implementations are provided. In addition, the container requires a dedicated Wrapper as an interface to communicate with the Backend Service. It provides functionality to receive the dataset, start the execution of the algorithm with provided parameters. Further, it collects statistics during execution and upon successful execution sends the results to the Backend Service.

Upon request from the Web Application, the Result Controller provides the learned CGM, stored in the Internal Database, for a comprehensive exploration and evaluation of the experimental results. The Web Application enables to examine the statistics and calculated metrics, e.g., the SHD, in case that ground truth is available for the observational dataset. Moreover, if the ground truth is not available the provided sampling opportunities from the observational dataset enable a robustness examination [21].

In summary, the presented architectural blueprint enables an extensive comparison of implementations for CSL with regard to runtime and accuracy of the learned causal structures.

\subsection{MPCSL- A Reference Implementation}

Based on the previously introduced architectural blueprint that covers the requirements on the pipeline for CSL in Figure 2, we provide a reference implementation called. The Web Application in

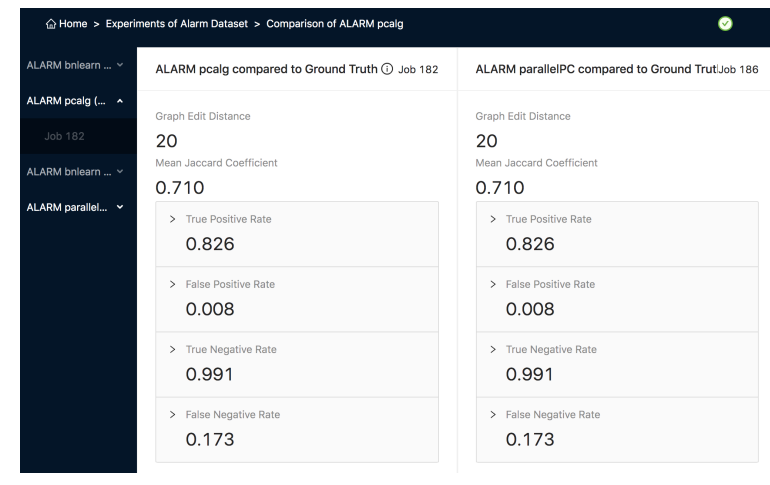

Figure 3: The Web Application of MPCSL provides an interface to the pipeline's components. For example, it enables to examine the accuracy metrics such as the Graph Edit Distance or Mean Jaccard Coefficient of identical graphs learned with pcalg (left) and parallelpC (right).

MPCSL is written in JavaScript using the React, Redux and D3 libraries. As shown in the example view in Figure 3, it provides an interface to interact with the different components of the pipeline necessary for an experimental evaluation.

It includes components to manage experiments, to compare and evaluate individual experiment runs, and to explore the learned causal model. The causal model explorer within the web application presents the learned relationships between variables, using a graph representation of edges for the relationships and nodes for the variables. It displays the original data distribution for each variable and supports functionality to estimate the causal effects of interventions following the do-operator paradigm.

The Backend Service of MPSCL is written in Python, using the Flask framework [12] to communicate with the Web Application through a RESTful API. In our reference implementation, the Dataset Manager uses SQL to communicate with the Internal Database an instance of a PostgreSQL [28] database and to communicate to external database management systems. We use the Python SQL toolkit and the object-relational mapper SQLAlchemy [2] to realize the communication and restrict the external data sources to those supported by SQLAlchemy.

The Experiment Execution Service uses the Python docker package to create individual docker containers for the execution of experiment runs, with the corresponding requirements of the experiment. The Execution Containers are based on Docker [26]. Currently, MPCSL provides docker images that support the R language [33], Python and that support the CUDA framework, when running on systems which include NVIDIA Graphics Processing Unit (GPU) hardware. Through the R execution environment, we support the packages pcalg [19], ParallelPC [24] and bnlearn [39]. With the CUDA execution environment, we added support for GPUaccelerated implementations [13, 38, 48].

For each experiment run, MPCSL collects and presents a selection of statistics, e.g., the runtime, or the number of learned edges. Moreover, the Result Controller provides the common accuracy metrics, such as the SHD or false positive rates of the learned causal model with respect to edges within the ground truth CGM. The 
examination of accuracy metrics, such as the graph edit distance or mean Jaccard coefficient, for differently learned CGMs enables a direct comparison of experiments on the same dataset, compare Figure 3, and, in combination with sampling, provides the basis for a robustness examination [21].

All implemented MPCSL services run in separate containers. The implementation uses Kubernetes to orchestrate the containers and execute them on different physical host systems, depending on the requirements of the CSL implementation.

\section{CASE STUDY ON RUNTIME OF ALGORITHMS FOR CSL USING MPCSL}

As previously introduced, data scientists trying to apply methods of CSL in real-world scenarios are confronted with a wide spectrum of different implementations. Their exists work that evaluates the different CSL approaches with respect to the accuracy of learned causal models, e.g., see [16, 41]. While this may support a data scientist in the selection of the appropriate approach, there is, to the best of our knowledge, no work that evaluates the runtime of different implementations on datasets with different data distributions. Especially medical scenarios, such as genetics, require fast implementations that allow for CSL in these high-dimensional settings $[1,19]$. As different implementations are written in different programming languages and utilize different hardware setups an evaluation with regard to runtime becomes a tedious manual task with high setup costs.

In the following case study, we illustrate the capabilities of MPCSL when comparing a selection of constraint-based CSL implementations with respect to their runtime. We choose constraint-based CSL for the following reasons. First, it is widely used in real-world scenarios $[18,24]$ making it an interesting case for practitioners. Second, it provides flexibility to adjust to different data distributions and dependencies by changing the utilized CI test accordingly. Third, constraint-based CSL implementations are available in many different programming languages, libraries, and packages. In particular, we focus on the well-known PC-Algorithm [44], especially its order-independent version PC-Stable [5]. Existing implementations with varying hardware acceleration of the PCStable $[13,19,22,24,36-39,48]$ are based upon the same algorithm, hence, should have equal accuracy for identically selected parameters, e.g., see Figure 3. Yet, specific implementation details impact the runtime, making it an interesting case for an experimental evaluation.

Our case study consists of three separate experiments on datasets with different data distributions. Further, the datasets differ in the number of variables $|V|$ and the number of observations $|N|$. Both characteristics impact the runtime of the PC-Stable algorithm. First, we conduct multiple experiment runs examining single-threaded runtimes of two different CSL implementations to provide an indication of the suitability in the context of given data distribution with the corresponding $\mathrm{CI}$ test, provided by the respective library. In a second and third experiment, we examine the potential of modern hardware to reduce the runtime of CSL. Therefore, we consider the speed-up of CSL implementations on a multi-core server and investigate the runtime of a GPU-accelerated implementation.

\begin{tabular}{ccrrc}
\hline Dataset & Distribution & $|V|$ & $|N|$ & Dimension \\
\hline NCI-60 & Gaussian & 1,190 & 47 & High \\
MCC & Gaussian & 1,380 & 88 & High \\
BR51 & Gaussian & 1,592 & 50 & High \\
DREAM5-INSILICO & Gaussian & 1,643 & 850 & High \\
S. AUREUS & Gaussian & 2,810 & 160 & High \\
S.CEREVISIAE & Gaussian & 5,361 & 63 & High \\
\hline ARTH150 & Gaussian & 107 & 20,000 & Low \\
ALARM & Discrete & 37 & 10,000 & Low \\
ANDES & Discrete & 223 & 20,000 & Low \\
LINK & Discrete & 724 & 20,000 & High \\
MUNIN & Discrete & 1,041 & 20,000 & High \\
MEHRA & Mixed & 24 & 20,000 & Low \\
\hline
\end{tabular}

Table 1: Characteristics of gene expression datasets and of datasets from the bnlearn repository. $|V|$ - number of vertices, $|N|$ - number of observations.

\subsection{Experimental Setup}

In the following, we provide an overview of the datasets, the hardware setup, and the CSL implementations supported by MPCSL for the experiments of our case study. During the setup of MPCSL we added the datasets as described below. We use the Web Application to parameterize the experiments for each CSL implementation. The experiment execution on the chosen hardware setup is orchestrated by the MPCSL Experiment Execution Service. After execution of multiple experiment runs, the requested metric, for our case study the median runtime, is presented in the Web Application.

Data: The characteristics of the datasets loaded into MPCSL for the experimental evaluation are provided in Table 1 . The datasets are either real-world gene expression datasets following a multivariate gaussian distribution, used in existing evaluations [24,38,48], or, generated datasets according to the well-known reference Bayesian networks from the bnlearn [39] network repository. The datasets contain different numbers of variable, ranging from, e.g., $|V|=24$ in MEHRA up to $|V|=5,361$ in S. CEREVISIAE. Thus, the datasets cover both low-dimensional datasets, which we define as $|V|<500$, and high-dimensional datasets, i.e., $|V| \geq 500$. Furthermore, the datasets incorporate different data distributions, for instance multivariate normal distributed data, called Gaussian, multinomial distributed data, called Discrete, and conditional Gaussian data, called Mixed.

Hardware Setup: All experiment runs are executed in a Kubernetes cluster consisting of a virtual machine, running the MPCSL Experiment Execution Service, the MPCSL Backend Service, including the Internal Database and an enterprise-grade server with 2 Intel ${ }^{\circledR}$ Xeon $^{\circledR}$ Gold $6148 \mathrm{CPU}$ with 20 cores each. The server is equipped with $1.5 \mathrm{~TB}$ of RAM, allowing to keep all data in memory during the execution of the experiment runs, and has 4 NVIDIA V100 card, with 32 GB of high bandwidth memory. The Execution Containers are run on the server. Furthermore, datasets are fetched from an external database management system running on a separate system. During measurements of the runtime, only a single container is executed at a time and no other operations are executed on the server. 
Measurements are conducted within the MPCSL Wrapper, measuring the algorithm's function call, e.g., for R library bnlearn [39] using the difftime of the Sys.time() prior to and after the call of pc.stable. If not stated differently, we repeat each experiment run at least 10 times and present the median runtime. Further, we set the tuning parameter $\alpha$ to 0.01 , which is common in application [5].

Employed CSL Implementations: We compare implementations of the PC-stable [5] from the well-known libraries pcalg [19] and bnlearn [39], as well as, from the libraries Parallelpc [24] and cupc [48], which allow for a comparison of different parallel execution strategies, and hardware acceleration in GPU-accelerated environments. The four implementations support Gaussian data using a Fisher's $Z$ test. For Discrete data bnlearn uses Pearson's $X^{2}$ test, pcalg and Parallelpc use the very similar $G^{2}$ test and cupc has no test implemented. Mixed data is only supported by bnlearn that incorporates a mutual information-based CI test for conditional Gaussian settings, provided through the mi-cg implementation. Parallelpc is written entirely in $\mathrm{R}$ and uses the $\mathrm{R} \mathrm{li-}$ brary parallel for parallel execution. Bnlearn is written in $\mathrm{R}$ and $\mathrm{C}$, using $\mathrm{C}$ to provide efficient implementations for most functions. It also uses the R library parallel for parallel execution. Pcalg is written in $\mathrm{R}$ and implements the test for Gaussian data in $\mathrm{C}++$. For this particular case it uses openMP [7] for parallel execution. In contrast to the previous libraries, cupc targets a heterogeneous system with an NVIDIA GPU. It provides an R Interface, yet the core algorithm is written using the CUDA framework [27] to utilize the parallel computing capabilities of a GPU.

\subsection{Single-Threaded Runtime}

In the following, we measure the single-threaded runtime of the PC-Stable implementation from pcalg and bnlearn on all datasets presented in Table 1. The measured runtimes are shown in Table 2.

For Gaussian datasets, our measurements show that the implementation from the pcalg library outperforms the bnlearn implementation by factors of 30 for low-dimensional datasets, e.g., ARTH150 and factors of up to 6,753 for high-dimensional datasets. For the datasets S. AUREUS and S. CEREVISIAE we terminated the execution of bnlearn after 5 days, without any result, whereas pcalg's implementation finished after 180 seconds. Both implementations use Fisher's $Z$ test for CI testing and are written in C or $\mathrm{C}++$. Yet, pcalg works on a pre-calculated correlation matrix, whereas within bnlearn the correlation is calculated repeatedly for each independence test, which we account for the significant difference in measured runtime. Note, the calculation of the correlation matrix prior to the execution of the PC-Stable, for the datasets subject of the study ranges from 0.044 seconds for NCI-60 to 1.391 seconds for S.CEREVISIAE. Thus, it does not impact the total runtime in a significant way.

For Discrete datasets the implementation from the bnlearn package outperforms pcalg implementation by factors of up to 25 , for low-dimensional datasets, e.g., ALARM, to factors of up to 84 for higher-dimensional datasets, e.g., LINK. For the dataset MUNIN the execution of the pcalg implementation was terminated after 5 days, without any result, compared to bnlearn's implementation, which finished below 2 hours. We account the difference to the fact

\begin{tabular}{crr}
\hline Dataset & pcalg & bnlearn \\
\hline ARTH150 & 248.7 & 7,617 \\
NCI-60 & 6.7 & 45,251 \\
MCC & 21.5 & 83,886 \\
BR51 & 32.3 & 171,629 \\
DREAM5-INSILICO & 4,257 & 360,572 \\
S. AUREUS & 180.0 & $>432,000$ \\
S. CEREVISIAE & 108.2 & $>432,000$ \\
\hline ALARM & 30.1 & 0.5 \\
ANDES & 2,824 & 108.8 \\
LINK & 324,873 & 3,827 \\
MUNIN & $>432,000$ & 6,753 \\
\hline MEHRA & - & 11.9
\end{tabular}

Table 2: Median runtimes in seconds of PC-stable in single threaded execution. Comparing implementations from libraries pcalg and bnlearn on benchmark datasets. Note, for algorithms exceeding the runtime of a day we executed a single run or terminated after five days.

that the CI test for Discrete data in pcalg is written in R, whereas bnlearn uses efficient $C$ code.

For the Mixed dataset MEHRA we report numbers only for bnlearn, as no other library used in the case study supports Mixed data. Based on the measurements, we conclude that it is advisable to use the implementation from the pcalg library for Gaussian data, whereas for Discrete and Mixed data the bnlearn library should be used.

\subsection{Parallel Efficiency on Multi-Core Systems}

In the following experiment, we measure the runtime of parallel implementations of the PC-Stable algorithm, taken from the libraries pcalg, Parallelpc and bnlearn. In previous studies $[24,40]$ the benefit of parallel execution to reduce the runtime of constraintbased CSL algorithms is shown, yet to the best of our knowledge no comparison between library-specific implementations exists. Therefore, we aim to determine differences in the implementations' behavior, when scaling the number of cores. All three implementations follow the framework for parallel constraint-based learning [40]. Yet, parallel processing within the implementations of the PC-Stable is applied only to the adjacency search, which comprises the CI tests. Other parts of the implementations are executed sequentially, which limits the effect of scaling the number of cores on the measured runtime.

In Table 3, we provide our measurements for the Gaussian dataset ARTH150 using all three libraries and for Discrete datasets using Parallelpc and bnlearn, given that pcalg does not support parallel execution for Discrete data. For all measurements, we observe that the ideal speed-up is not achieved, which is due to the sequential part of the implementations. For the dataset ARTH150, Parallelpc shows the implementations' worst speed-up, achieving only a factor of up to 7.15 on 40 cores compared to a factor 15.51 speed-up of pcalg. Except for this particular difference, the behavior is similar for all implementations. Note, the overall runtime remains significantly slower for bnlearn compared to pcalg. 


\begin{tabular}{llrrrr}
\hline Library & Dataset & \multicolumn{4}{c}{ Cores } \\
& & 5 & 10 & 20 & 40 \\
\hline Parallelpc & ARTH150 & 3.3 & 4.1 & 6.9 & 7.2 \\
bnlearn & ARTH150 & 3.3 & 5.3 & 8.3 & 11.7 \\
pcalg & ARTH150 & 3.0 & 5.7 & 8.9 & 15.5 \\
\hline Parallelpc & ANDES & 3.4 & 6.2 & 10.9 & 14.3 \\
bnlearn & ANDES & 1.6 & 1.6 & 1.6 & 1.4 \\
bnlearn & LINK & 3.4 & 5.3 & 7.0 & 7.6 \\
bnlearn & MUNIN & 4.0 & 6.8 & 11.3 & 14.4 \\
\hline
\end{tabular}

Table 3: Factors of speed-up measured with an increasing number of cores running parallel versions of the PC-Stable compared to single threaded execution.

\begin{tabular}{crr}
\hline Dataset & cupc & pcalg (40 cores) \\
\hline ARTH150 & 1.93 & 16.03 \\
NCI-60 & 1.94 & 3.18 \\
MCC & 2.45 & 4.93 \\
BR51 & 4.48 & 6.42 \\
DREAM5-INSILICO & 5.41 & 168.09 \\
S. AUREUS & 13.58 & 35.21 \\
S.CEREVISIAE & 32.79 & 55.59 \\
\hline
\end{tabular}

Table 4: Median runtime in seconds of $P C$-stable with the adjacency search executed on a GPU - cupc or in parallel using 40 CPU cores - pcalg.

Considering Discrete datasets, we observe a runtime improvement for bnlearn using up to 40 cores for the high-dimensional datasets LINK and MUNIN. For the dataset ANDES, speed-up through the increase in cores is small with factors below 2 and even decreases when running on 40 cores compared to 20 cores. We assume that the sequential part of the execution dominates the processing of the dataset ANDES in bnlearn and that the adjacency search is already efficient in single-threaded execution. In contrast processing ANDES with ParallelPC, we observe strong speed-up, yet the overall runtime for 40 cores is slower than the single-threaded execution with bnlearn. This supports our assumption that bnlearn has efficient adjacency search and CI test implementations for Discrete data.

Overall, we did not observe a significant difference in the implementations' behavior with regard to scaling the number of cores. Yet, for lower-dimensional datasets, e.g., ANDES, efficient implementations, e.g., found in bnlearn, cannot benefit from multi-core systems and may even be slowed down.

\subsection{GPU-Acceleration}

In a third experiment, we consider a heterogeneous system and investigate the speed-up of a GPU-accelerated implementation, cupc, over a Central Processing Unit (CPU)-based parallel implementation, pcalg running on 40 cores. The MPCSL Experiment Execution Service ensures that the experiment runs are scheduled on hardware that fulfills the requirements to execute the implementations, e.g., provide an NVIDIA GPU for cupc.
In Table 4, we report the measured runtimes on all Gaussian datasets considered in our case study. Using a GPU to process the datasets leads to additional speed-up for all datasets, ranging from speed-up of factor 1.43 for dataset BR51 to speed-up of factor 31.07 for dataset DREAM5-INSILICO. We observe that the two datasets, DREAM5-INSILICO and ARTH150 result in higher speed-up when the adjacency search is executed on the GPU. Both datasets have a larger number of observations $|N|$, compared to the other datasets, which results in an increased number of higher-order CI tests. For the other five datasets, execution on the GPU leads to a speed-up of less than factor 2 . For these datasets, over $94 \%$ of the independence tests are either unconditioned or with a conditioning set of size 1 [38] for the given tuning parameter $\alpha=0.01$. We account for the high percentage of low-order independence tests for the small factor of speed-up achievable with GPU-acceleration.

\section{SUMMARY}

In our work, we present a blueprint and a reference implementation of a modular pipeline for Causal Structure Learning (CSL). The pipeline supports the experimental evaluation of the wide spectrum of implementations of algorithms for CSL from data scientists and their application in practice. In particular, the platform manages the observational datasets and parameter setups for experiment runs. Further, it orchestrates the execution of the experiments with given hardware constraints using virtualized environments. Storing the parameterization ensures reproducibility. The collection of runtime statistics, and accuracy metrics allows for a comprehensive experimental evaluation in the pipeline.

We present the capabilities of our reference implementation MPCSL that was developed to address the requirements of deployment in scenarios from both a theoretical and practical perspective. In detail, we conduct a case study using MPCSL to investigate the runtime performance of implementations of the well-known PC-Algorithm [44]. In this scenario, the results indicate that for Gaussian data, the pcalg library provides better runtimes, by factors of over 1,000, whereas for Discrete data the bnlearn library outperforms the pcalg implementation by factors of up to 84 . An investigation of parallel execution on multi-core systems showed similar behavior with an increasing number of cores across the implementations, achieving a speed-up of up to factor 15.5 on 40 cores. Additional speed-up of factors of up to 31 is possible employing GPUs, yet their application is currently limited to Gaussian data.

Beyond the demonstrated research aspect, we use the developed tool with industry partners from the manufacturing domain. Here, the focus is on comparing the accuracy of learned CGMs with different approaches for CSL, as well as, different data preparation techniques. In this context, we found that there is a strong need for visual support, during the selection and preparation of data for CSL, and when comparing different learned CGMs. Currently, the reference implementation is limited to showing a single CGM and only reports accuracy metrics when comparing multiple CGMs.

In future work, we are going to incorporate the opportunity to generate datasets with different data characteristics following a selection of data-generating models such that the pipeline envelopes the whole benchmarking pipeline. While MPCSL currently serves as a starting point, the pipeline's modularity with respect to the CTF 
enables for extensibility regarding the causally insufficient case or interventional data. In this context, we aim to extend the platform to directly provide a larger set of CSL implementations to further raise the interest of the research community. We invite the research community to actively participate in the extension of MPCSL.

\section{REFERENCES}

[1] Michael Banf and Seung Y Rhee. 2017. Computational inference of gene regulatory networks: Approaches, limitations and opportunities. Biochimica et Biophysica Acta (BBA)-Gene Regulatory Mechanisms 1860, 1 (2017), 41-52.

[2] Michael Bayer. 2012. SQLAlchemy. In The Architecture of Open Source Applications Volume II: Structure, Scale, and a Few More Fearless Hacks. aosabook.org.

[3] David Maxwell Chickering. 2003. Optimal Structure Identification with Greedy Search. Journal of Machine Learning Resesarch 3 (March 2003), 507-554.

[4] David Maxwell Chickering, David Heckerman, and Christopher Meek. 2004 Large-Sample Learning of Bayesian Networks is NP-Hard. Fournal of Machine Learning Research 5 (Dec. 2004), 1287-1330.

[5] Diego Colombo and Marloes H. Maathuis. 2014. Order-independent Constraintbased Causal Structure Learning. Journal of Machine Learning Research 15, 1 (Jan. 2014), 3741-3782.

[6] Stefan Conrady and Lionel Jouffe. 2015. Bayesian Networks and BayesiaLab: A Practical Introduction for Researchers. Bayesia USA, Franklin, USA. 382 pages.

[7] Leonardo Dagum and Ramesh Menon. 1998. OpenMP: An Industry-Standard API for Shared-Memory Programming. IEEE Computational Science and Engineering 5, 1 (Jan. 1998), 46-55.

[8] David Donoho. 2017. 50 Years of Data Science. Journal of Computational and Graphical Statistics 26, 4 (2017), 745-766.

[9] Vincent Dorie, Jennifer Hill, Uri Shalit, Marc Scott, and Dan Cervone. 2019. Automated versus Do-It-Yourself Methods for Causal Inference: Lessons Learned from a Data Analysis Competition. Statist. Sci. 34, 1 (02 2019), 43-68.

[10] Frank Emmert-Streib, Galina Glazko, Ricardo De Matos Simoes, et al. 2012. Statistical inference and reverse engineering of gene regulatory networks from observational expression data. Frontiers in Genetics 3 (2012), 8

[11] Clark Glymour, Kun Zhang, and Peter Spirtes. 2019. Review of Causal Discovery Methods Based on Graphical Models. Frontiers in genetics 10 (2019), 524

[12] Miguel Grinberg. 2014. Flask Web Development: Developing Web Applications with Python (1st ed.). O'Reilly Media, Inc., Sebastopol, CA, USA.

[13] Christopher Hagedorn and Johannes Huegle. 2021. GPU-Accelerated ConstraintBased Causal Structure Learning for Discrete Data. In Proceedings of the 2021 SIAM International Conference on Data Mining (SDM). Society for Industrial and Applied Mathematics, Philadelphia, PA, USA, 37-45.

[14] Alain Hauser and Peter Bühlmann. 2012. Characterization and Greedy Learning of Interventional Markov Equivalence Classes of Directed Acyclic Graphs. Journal of Machine Learning Research 13, 1 (Aug. 2012), 2409-2464.

[15] David Heckerman, Dan Geiger, and David M. Chickering. 1995. Learning Bayesian Networks: The Combination of Knowledge and Statistical Data. Machine Learning 20, 3 (01 Sep 1995), 197-243.

[16] Christina Heinze-Deml, Marloes H. Maathuis, and Nicolai Meinshausen. 2018 Causal Structure Learning. Annual Review of Statistics and Its Application 5, 1 (2018), 371-391.

[17] Miguel A Hernán et al. 2019. Comment: Spherical Cows in a Vacuum: Data Analysis Competitions for Causal Inference. Statist. Sci. 34, 1 (2019), 69-71.

[18] Johannes Huegle, Christopher Hagedorn, and Matthias Uflacker. 2020. How Causal Structural Knowledge Adds Decision-Support in Monitoring of Automotive Body Shop Assembly Lines. In Proceedings of the Twenty-Ninth International foint Conference on Artificial Intelligence, IfCAI-20. IJCAI Organization, Yokohama, 5246-5248.

[19] Markus Kalisch, Martin Mächler, Diego Colombo, Marloes H. Maathuis, and Peter Bühlmann. 2012. Causal Inference Using Graphical Models with the R Package pcalg. Fournal of Statistical Software, Articles 47, 11 (2012), 1-26.

[20] Ehud Karavani, Tal El-Hay, Yishai Shimoni, and Chen Yanover. 2019. Comment: Causal Inference Competitions: Where Should We Aim? Statist. Sci. 34, 1 (2019), 86-89.

[21] Erich Kummerfeld and Alexander Rix. 2019. Simulations evaluating resampling methods for causal discovery: ensemble performance and calibration. In 2019 IEEE International Conference on Bioinformatics and Biomedicine, BIBM 2019. IEEE, San Diego, CA, USA, 2586-2593.

[22] Vincenzo Lagani, Giorgos Athineou, Alessio Farcomeni, Michail Tsagris, and Ioannis Tsamardinos. 2017. Feature Selection with the R Package MXM: Discovering Statistically Equivalent Feature Subsets. Fournal of Statistical Software 80, 7 (2017), 1-25.

[23] Thuc Duy Le, Tao Hoang, Jiuyong Li, Lin Liu, Huawen Liu, and Shu Hu. 2019. A Fast PC Algorithm for High Dimensional Causal Discovery with Multi-Core PCs. IEEE/ACM Transactions on Computational Biology and Bioinformatics 16, 5 (2019),
1483-1495.

[24] Thuc Duy Le, Taosheng Xu, Lin Liu, Hu Shu, Tao Hoang, and Jiuyong Li. 2018. ParallelPC: An R Package for Efficient Causal Exploration in Genomic Data. In Pacific-Asia Conference on Knowledge Discovery and Data Mining. Springer International Publishing, Cham, 207-218.

[25] Alexander Lin, Amil Merchant, Suproteem K. Sarkar, and Alexander D'Amour. 2019. Universal Causal Evaluation Engine: An API for Empirically Evaluating Causal Inference Models. In Proceedings of Machine Learning Research, Vol. 104. PMLR, Anchorage, Alaska, USA, 50-58

[26] Dirk Merkel. 2014. Docker: Lightweight Linux Containers for Consistent Development and Deployment. Linux fournal 2014, 239 (March 2014).

[27] John Nickolls, Ian Buck, Michael Garland, and Kevin Skadron. 2008. Scalable Parallel Programming with CUDA. Queue 6, 2 (March 2008), 40-53.

[28] Regina Obe and Leo Hsu. 2012. PostgreSQL: Up and Running. O’Reilly Media, Inc., Sebastopol, CA, USA.

[29] Juan Miguel Ogarrio, Peter Spirtes, and Joe Ramsey. 2016. A hybrid causal search algorithm for latent variable models. In Conference on Probabilistic Graphical Models, Vol. 52. PMLR, Lugano, Switzerland, 368-379.

[30] D. L. Parnas. 1972. On the Criteria to Be Used in Decomposing Systems into Modules. Commun. ACM 15, 12 (Dec. 1972), 1053-1058.

[31] Judea Pearl. 2009. Causality: Models, Reasoning and Inference (2nd ed.). Cambridge University Press, New York, NY, USA.

[32] Jonas Peters, Dominik Janzing, and Bernhard Schölkopf. 2017. Elements of Causal Inference - Foundations and Learning Algorithms. MIT Press, Cambridge, MA, USA. 288 pages.

[33] R Core Team. 2017. R: A Language and Environment for Statistical Computing. R Foundation for Statistical Computing, Vienna, Austria.

[34] Andrea Rau, Florence Jaffrézic, and Grégory Nuel. 2013. Joint Estimation of Causal Effects from Observational and Intervention Gene Expression Data. BMC systems biology 7, 1 (Oct 2013), 111.

[35] Dominik Rothenhäusler, Christina Heinze, Jonas Peters, and Nicolai Meinshausen. 2016. BACKSHIFT: Learning Causal Cyclic Graphs from Unknown Shift Interventions. In Advances in Neural Information Processing Systems 28. Curran, Red Hook, NY, 1513-1521.

[36] Richard Scheines, Peter Spirtes, Clark Glymour, Christopher Meek, and Thomas Richardson. 1998. The TETRAD Project: Constraint Based Aids to Causal Model Specification. Multivariate Behavioral Research 33, 1 (1998), 65-117.

[37] Christopher Schmidt, Johannes Huegle, Philipp Bode, and Matthias Uflacker. 2019. Load-Balanced Parallel Constraint-Based Causal Structure Learning on MultiCore Systems for High-Dimensional Data. In Proceedings of Machine Learning Research, Vol. 104. PMLR, Anchorage, Alaska, USA, 59-77.

[38] Christopher Schmidt, Johannes Huegle, and Matthias Uflacker. 2018. Orderindependent Constraint-based Causal Structure Learning for Gaussian Distribution Models Using GPUs. In Proceedings of the 30th International Conference on Scientific and Statistical Database Management (Bozen-Bolzano, Italy) (SSDBM '18). ACM, New York, NY, USA, Article 19, 10 pages.

[39] Marco Scutari. 2010. Learning Bayesian Networks with the bnlearn R Package. Journal of Statistical Software, Articles 35, 3 (2010), 1-22.

[40] Marco Scutari. 2017. Bayesian Network Constraint-Based Structure Learning Algorithms: Parallel and Optimized Implementations in the bnlearn R Package. Journal of Statistical Software, Articles 77, 2 (2017), 1-20.

[41] Marco Scutari, Catharina Elisabeth Graafland, and José Manuel Gutiérrez. 2018. Who Learns Better Bayesian Network Structures: Constraint-Based, Score-based or Hybrid Algorithms? In Proceedings of the Ninth International Conference on Probabilistic Graphical Models, Vol. 72. PMLR, Prague, Czech Republic, 416-427.

[42] Shohei Shimizu, Patrik O. Hoyer, Aapo Hyvärinen, and Antti Kerminen. 2006. A Linear Non-Gaussian Acyclic Model for Causal Discovery. Fournal of Machine Learning Research 7 (Dec. 2006), 2003-2030.

[43] Yishai Shimoni, Chen Yanover, Ehud Karavani, and Yaara Goldschmnidt. 2018. Benchmarking Framework for Performance-Evaluation of Causal Inference Analysis. arXiv:1802.05046 [stat.ME]

[44] Peter Spirtes, Clark N Glymour, and Richard Scheines. 2000. Causation, Prediction, and Search. MIT press, Cambridge, MA, USA.

[45] Robert E. Tillman, Arthur Gretton, and Peter Spirtes. 2009. Nonlinear Directed Acyclic Structure Learning with Weakly Additive Noise Models. In Proceedings of the 22nd International Conference on Neural Information Processing Systems (NIPS'09). Curran Associates Inc., Red Hook, NY, USA, 1847-1855.

[46] Michail Tsagris. 2019. Bayesian Network Learning with the PC Algorithm: An Improved and Correct Variation. Applied Artificial Intelligence 33, 2 (2019), 101123

[47] Ioannis Tsamardinos, Laura E. Brown, and Constantin F. Aliferis. 2006. The maxmin hill-climbing Bayesian Network Structure Learning Algorithm. Machine Learning 65, 1 (01 Oct 2006), 31-78.

[48] Behrooz Zarebavani, Foad Jafarinejad, Matin Hashemi, and Saber Salehkaleybar. 2019. cuPC: CUDA-based Parallel PC Algorithm for Causal Structure Learning on GPU. IEEE Transactions on Parallel and Distributed Systems (TPDS) 31, 03 (2019), $530-542$. 